\title{
A BLIND JOINT ESTIMATOR FOR MULTIPATH DIVERSITY AND PN TIMING ERROR IN DIRECT-SEQUENCE SPREAD-SPECTRUM RECEIVERS
}

\author{
Jia-Chin Lin \\ Department of Electrical Engineering, National Taiwan University Taipei, Taiwan
}

\begin{abstract}
A blind joint estimator for multipath diversity and PN code timing error is proposed in this paper for direct-sequence spread-spectrum signaling on a frequency-selective fading channel. In the multipath diversity combiner, a modified known modulus adaptive (KMA) algorithm is used to cope with time-varying multipath effects and to perform multipath diversity combining in the blind mode. In the code timing recovery, the timing error signal is extracted from each propagation path independently and also combined in the same fashion as the multipath diversity combining process. By taking advantage of the inherent diversity based on a known modulus adaptive (KMA) algorithm, this modified code timing recovery can avoid the problem due to the drift or flutter effects of the error signals, and provide better performance on frequency selective fading channels. Extensive computer simulation results have verified the analysis and indicated very attractive behavior of the proposed joint estimator for multipath diversity and PN code timing error.
\end{abstract}

\section{INTRODUCTION}

Frequency-selective fading can often lead to severe performance degradation in wideband communication systems. Substantial efforts have been made in adaptive equalization and diversity combining (RAKE) techniques in order to improve overall receiver performance [1]. However, only when both multipath diversity combining and code timing recovery are optimized simultaneously on the frequency-selective fading channel, can overall receiver performance be improved. In this paper, a blind joint estimator for multipath diversity and code timing error is proposed. Based on this technique, a KMA-based multipath diversity combiner operates in conjunction with a modified code timing recovery to achieve simultaneous improvement of overall receiver performance with lower computation load and lower processing rate. The modified KMA exploits a nonlinear step-size parameter to achieve better stability and improved robustness with noise and time-varying channel to accomplish better blind multipath diversity combining. In the code timing recovery derived from the PN code timing error estimator, the timing error signal is extracted from each propagation path independently and also combined in the same fashion as the multipath diversity combining process. Due to the inherent diversity based on the modified KMA technique, this modified code timing recovery can avoid the problem caused by the drift or flutter effects of the error signals [2], and provide better code tracking performance. Therefore, the proposed technique can improve the overall receiver performance.

\section{DESCRIPTION OF THE PROPOSED BLIND JOINT ESTIMATOR}

To describe in detail the operations in the RAKE receiver, which is based on the proposed blind joint estimator for multipath diversity and the PN code timing error, a complete block diagram of the RAKE receiver is sketched in Fig. 1. The complex representation of the baseband signal at the output of the chip matched filter is

$$
\tilde{r}(t)=\sum_{n=0}^{L-1} a_{n}(t) s\left(t-n T_{c}\right)+n(t)
$$

where $s(t)=\sum_{i=0}^{\infty} d_{i} \sum_{l=0}^{M-1} c_{l} g\left(t-l T_{c}-i T_{b}\right)$ is the datamodulated PN sequence with raised cosine chip shaping, $a_{n}(t)$ are the time-varying complex-valued tap weights with Rayleigh distributed magnitudes and uniformly distributed phases, $L$ is the number of resolvable paths, $d_{i}$ is the $\mathrm{i}$-th information-bearing symbol, $c_{l}$ is the $l$-th chip value of the $\mathrm{PN}$ sequence, $T_{b}$ and $T_{c}$ are the symbol interval and chip duration respectively, $M=\frac{T_{b}}{T_{c}}$ is the processing gain, $g(t)$ is the overall chip shape. The effect of carrier phase error introduced by the front-end noncoherent down conversion process is absorbed into the time-varying channel weights, $a_{n}(t)$. Without loss of generality, it is assumed for simplicity here the period of the PN code is exactly $M$.

II-A. Multipath Diversity Combining Technique The integer-instant sample stream

$$
\tilde{r}_{k}=\sum_{n=0}^{L-1} a_{n}\left(k T_{c}\right) s\left(k T_{c}-n T_{c}+\varepsilon_{k} T_{c}\right)+n\left(k T_{c}\right)
$$

is fed into the KMA-based multipath diversity combiner. Assume first the code acquisition process has been achieved. In the each arm of the multipath diversity combiner, the input samples, $\tilde{r}_{k-m}, \quad m=0,1, \cdots, L-1$, are crosscorrelated with the local PN sequence $c_{k-(L-1)}$, which 
has been code-acquired, and then pass through the arm filter, $h_{k}$, which is lowpass filter with the bandwidth comparable with the symbol rate and its transfer function, $H(z)=\frac{1-a}{1-a z^{-1}}$, to produce the KMA input samples,

$$
\begin{aligned}
x_{k}^{m}= & \tilde{r}_{k-m} \times c_{k-(L-1)} * h_{k} \\
= & a_{(L-1)-m} g\left(\varepsilon_{k}\right) \sum_{i=0}^{\infty} d_{i} \cdot D_{k-i M}+S . N .+n_{k-m}^{\prime} \\
& m=0,1, \cdots, L-1 .
\end{aligned}
$$

where $x$ denotes correlation operator, $*$ denotes the convolution operator, $D_{k}=\sum_{l=0}^{M-1} \delta(k-l) * h_{k}=(1-$ $\left.a^{k}\right)\left[u_{k}-u_{k-M}\right]+O_{1}$ for each one symbol duration, S.N. is caused by self-noise, $u_{k}$ denotes a discrete unit step function, $O_{1}$ is an undesired term which is the response outside one symbol duration and it can be eliminated by reseting the arm filter output at the beginning of each symbol period. To achieve the multipath diversity combining, a multipath diversity combiner with coefficient vector, $\boldsymbol{W}_{k}=\left[w_{k}^{0}, w_{k}^{1}, \cdots, w_{k}^{L-1}\right]^{T}$, is employed. The multipath diversity combiner output is

$$
y_{k}=\boldsymbol{W}_{k}^{T} \boldsymbol{X}_{k}
$$

where $\boldsymbol{X}_{k}=\left[x_{k}^{0}, x_{k}^{1}, \cdots, x_{k}^{L-1}\right]^{T}$ is the input vector of the KMA algorithm. In order to achieve blind RAKE combining together with carrier phase recovery, the cost function of the conventional KMA algorithm given as below

$$
J_{k}=E\left\{\left.|| y_{k}\right|^{2}-\left.R_{k}\right|^{2}\right\}
$$

must be written as $J_{k}=J_{k}^{R}+J_{k}^{I}$, where $J_{k}^{R}$ and $J_{k}^{I}$ are the cost functions for real and imaginary parts of the equalizer output, $y_{k}=y_{k}^{R}+j y_{k}^{I}$, and are defined as

$$
\left\{\begin{array}{l}
J_{k}^{R}=E\left[\left(\left(y_{k}^{R}\right)^{2}-R_{k}^{R}\right)^{2}\right] \\
J_{k}^{I}=E\left[\left(\left(y_{k}^{I}\right)^{2}-R_{k}^{I}\right)^{2}\right]
\end{array}\right.
$$

Assuming the transmitted data symbols, $d_{k}=d_{k}^{R}+j d_{k}^{I}$, are i.i.d. and sampled at the time instant $t=k T_{c}$, then $R_{k}^{R}$ and $R_{k}^{I}$ are determined by

$$
\left\{\begin{array}{l}
R_{k}^{R}=\frac{E\left\{\left[d_{k}^{R} * h_{k}\right]^{4}\right\}}{E\left\{\left[d_{k}^{R} * h_{k}\right]^{2}\right\}} \\
R_{k}^{I}=\frac{\left.E\left[d_{k}^{I} * h_{k}\right]^{4}\right\}}{E\left\{\left[d_{k}^{I} * h_{k}\right]^{2}\right\}}
\end{array}\right.
$$

for the real and imaginary parts of the lowpass filtering transmitted data symbols respectively. Because in the receiver the despreading process, i.e., cross-correlation with local code-acquired PN sequence, is followed by the lowpass arm filter, $h_{k}$, the desired multipath diversity combiner output is lowpassed and thus $R_{k}^{R}$ and $R_{k}^{I}$ defined above is determined by the lowpass data symbols, $d_{k}^{R} * h_{k}$ and $d_{k}^{I} * h_{k}$. With this modification, the modified KMA can not only combine the signals from each propagation path, but also track the carrier frequency offset to avoid random spinning of the output signal constellation. In accordance with [6], we rewrite the update procedure as follows,

$$
\boldsymbol{W}_{k+1}-\boldsymbol{W}_{k}=-\frac{\mu}{\left\|\boldsymbol{X}_{k}\right\|^{2}} \boldsymbol{X}_{k}^{*}\left[\hat{e}_{k}^{R}+j \hat{e}_{k}^{I}\right]
$$

, the error signal, $\left[\hat{e}_{k}^{R}+j \hat{e}_{k}^{I}\right]$, could thus be approximated as

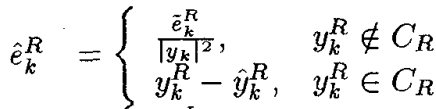

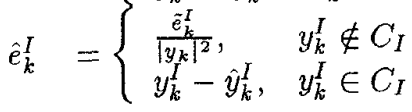

where

$$
\left\{\begin{array}{l}
\tilde{e}_{k}^{R}=y_{k}^{R}\left(\left(y_{k}^{R}\right)^{2}-R_{k}^{R}\right) \\
\tilde{e}_{k}^{I}=y_{k}^{I}\left(\left(y_{k}^{I}\right)^{2}-R_{k}^{I}\right)
\end{array}\right.
$$

and $C_{R}$ and $C_{I}$ are the confidence zones of real and imaginary parts respectively defined in [7]; $\hat{y}_{k}^{R}$ and $\hat{y}_{k}^{I}$ are the real and imaginary parts of the decision result of the multipath diversity combiner output. Initially, the combining process begins when the error is large, the modified KMA operates in the blind mode described as

$$
\boldsymbol{W}_{k+1}-\boldsymbol{W}_{k} \cong-\frac{\mu}{\left\|\boldsymbol{X}_{k}\right\|^{2}\left|y_{k}\right|^{2}+a} \boldsymbol{X}_{k}^{*}\left[\tilde{e}_{k}^{R}+j \tilde{e}_{k}^{I}\right]
$$

where $a$ is a small positive number included to avoid any possible divergence caused by $\left\|\boldsymbol{X}_{k}\right\|^{2}\left|y_{k}\right|^{2}=0$. Whenever the output error is low enough, the decision-directed algorithm with normalized LMS (NLMS) adaptation takes place in the tracking mode. The update equation is described as

$$
\boldsymbol{W}_{k+1}-\boldsymbol{W}_{k}=-\frac{\mu}{\left\|\boldsymbol{X}_{k}\right\|^{2}+b} \boldsymbol{X}_{k}^{*}\left[y_{k}-\hat{y}_{k}\right]
$$

where $b$ is a small positive value again and $\hat{y}_{k}$ is the decision result of the multipath diversity combiner output at time $k$. These two modes can be switched over automatically and smoothly according to the magnitude of the multipath diversity combiner output error without any specific detection mechanism.

In addition, if we define the step-size parameter as

$$
\hat{\mu}=\frac{\mu}{\left\|\boldsymbol{X}_{k}\right\|^{2}\left|y_{k}\right|^{2}+a}
$$

we may view the modified KMA algorithm proposed here as a KMA algorithm with a time-varying step-size parameter, $\hat{\mu}$. This time-varying step-size parameter can overcome the gradient noise amplification problem occurring with conventional estimation algorithms whenever the input vector $\boldsymbol{X}_{k}$ increases suddenly. Obviously, $\hat{\mu}$ is indeed identical to the Kalman filter gain with a fixed positive-definite matrix as the error covariance matrix to guarantee local stability in the blind-mode of the modified KMA algorithm. 


\section{II-B. PN Code Chip Timing Recovery}

In the previous subsection, the operation of the multipath diversity combiner is described as $y_{k}=\sum_{m=0}^{L-1} w_{k}^{m} x_{k}^{m}$. If the cost function of the conventional KMA algorithm, $J_{k}=E\left\{\left.|| y_{k}\right|^{2}-\left.R_{k}\right|^{2}\right\}$, where $R_{k}=\frac{E\left\{\left\|d_{k} * h_{k}\right\|^{4}\right\}}{E\left\{\left\|d_{k} * h_{k}\right\|^{2}\right\}}$, is employed for the timing error estimator, the PN code timing error, $\varepsilon_{k}$, can be estimated by the following equation $\varepsilon_{k+1}=\varepsilon_{k}-\gamma_{t}^{\prime} \operatorname{Re}\left\{y_{k}^{*}\left[\left|y_{k}\right|^{2}-R_{k}\right] \cdot\left[\sum_{m=0}^{L-1} w_{k}^{m} \dot{x}_{k}^{m}\right]\right\}$, where $\gamma_{t}^{\prime}$ is the step-size parameter and $\dot{x}_{k}^{m}$ is the differentiate value of $x(t)$ at the timing instant $t=(k-m) T_{c}$. For simplicity, $y_{k}\left[\left|y_{k}\right|^{2}-R_{k}\right]$ is replaced by the decision result of the multipath diversity combiner output, $\hat{y}_{k}$, and then the update equation can be rewritten as $\varepsilon_{k+1}=\varepsilon_{k}-\gamma_{t}^{\prime \prime} \operatorname{Re}\left\{\hat{y}_{k}^{*} \sum_{m=0}^{L-1} w_{k}^{m} \dot{x}_{k}^{m}\right\}$. Because of fulldigital implementation, the differentiate operation must be replaced by the difference operation. If we define $\dot{x}_{k}^{m}=\frac{1}{T_{c}}\left\{x_{k-\frac{1}{2}}^{m}-x_{k+\frac{1}{2}}^{m}\right\}$, we can implement the PN code timing recovery based on the timing error estimator described above in the same structure as that of the modified code tracking loop [5].

\section{SIMULATION RESULTS}

Computer simulation results illustrating the performance of the proposed blind joint estimator for multipath diversity and PN code timing error are presented in this section. The residual inter-chip interference (ICI) after the modified KMA proposed here, the timing error signals, mean square timing error and mean time to lose lock (MTLL) for the code timing recovery derived from the proposed PN code chip timing error estimator are evaluated by Monte Carlo methods. The simulation parameters are given below.

$\begin{array}{ll}\text { Modulation: } & \text { 4-QAM modulation } \\ \text { PN code: } & \text { m-sequence } \\ \text { Chip Shaping: } & \text { Square root raised cosine } \\ \text { Chip Rate: } & \frac{1}{T_{c}}=1.27 M \text { chips } / \mathrm{sec} \\ \text { Symbol Rate: } & \frac{1}{T_{b}}=10 \mathrm{~K} \text { symbols/sec } \\ \text { Sampling Rate: } & 16 \text { samples per chip period } \\ \text { Channel : } & 7 \text { tap weights are taken from [7], } \\ & \text { and each tap weight is corruptted }\end{array}$
and each tap weight is corruptted by an independent Jakes' fading maximal Doppler frequencies $8,80,10,100,25,30,35 \mathrm{~Hz}$

RAKE Structure: 7 arms with filters: $B_{b}=\frac{1}{T_{b}}$ Shown in Fig. 2-(a) is the signal constellation obtained after the multipath diversity combiner with the modified KMA algorithm proposed in this paper. It is obvious the proposed KMA-based combiner is qualified to perform multipath diversity combining and cluster the signal constellations at the right position very well. The residual inter-chip interference (ICI) at the output of the multipath diversity combiner is defined in the similar fashion of residual inter-symbol interference (ISI) [8] as follows:

$$
I C I=\frac{\sum_{k}\left[\left|h_{k} * w_{k}\right|^{2}\right]-\left|h_{k} * w_{k}\right|_{\text {max }}^{2}}{\left|h_{k} * w_{k}\right|_{\max }^{2}}
$$

where $\left|h_{k} * w_{k}\right|_{\max }$ is the maximum absolute value among all values of $h_{k} * w_{k}$. Fig. 2-(b) shows the ensemble average residual ICI after the KMA-based combiner proposed here from 100 Monte Carlo runs. Fig. 3 presents several short-period error signals of the proposed code timing recovery, the modified code tracking loop with the maximum ratio combining (MCTL/MRC) technique [5], and the conventional DLL. It is obvious from Figs. 3 -(a) \& (b) that the error signals of the proposed code timing recovery and MCTL/MRC [5] with stable locked points at $\varepsilon=0$, there exist no phenomena of drift or flutter of the locked point which are observed in Fig. 3-(c) for conventional DLLs [2]. Because of the timevarying channel, the received signals don't have constant strength. Thus, the strength of the error signal of the MCTL/MRC [5] shown in Fig. 3-(b) is timevarying. In performing KMA, the adaptive algorithm acts as an automatic gain control (AGC) in addition to its desired task of spectral shaping. The effect of AGC on the multipath diversity combiner is rejection of multiple access interference, while it also makes the error signals more stable and have the same strength shown in Fig. 3-(a). The mean square timing errors of the proposed code timing recovery, MCTL/MRC [5], and the conventional DLL under different SNR conditions are shown in Fig. 4-(a). We can see that the conventional DLL is vulnerable to multipath channel effect and suffers from an irreducible error floor. On the other hand, the proposed code timing recovery and MCTL/MRC [5] are with much less mean square timing errors than the conventional DLL. Moreover, the mean square timing errors of the proposed code timing recovery are less than that of MCTL/MRC [5]. It is believed that this is because the effect of AGC makes the error signals more steady (with the same strength) to avoid the misadjustment caused by variation of the error signals. Fig. 4-(b) presents the simulated results for the MTLL of the proposed code timing recovery, MCTL/MRC [5] and the conventional DLL under different SNR conditions. We can see that the proposed code timing recovery and MCTL/MRC [5] have much longer MTLL than the conventional DLL. Furthermore, the proposed code timing recovery has a longer MTLL than MCTL/MRC [5]. This is because the larger area, in average, under the error signals of the proposed code timing recovery for positive $\varepsilon$ implies higher escape energy, which can support longer MTLL.

\section{CONCLUSION}

A blind joint estimator for multipath diversity and PN code timing error is proposed in this paper. It has been shown that this technique can cope with time-varying 
multipath effects to accomplish multipath diversity combining in blind mode. By exploiting the multipath diversity, the code timing recovery provides better performance on frequency-selective fading channels in the sense of mean square timing error and MTLL.

\section{ACKNOWLEDGEMENT}

I am deeply indebted to my advisor, Prof. Lin-Shan Lee, for making so many valuable suggestions to improve this paper.

\section{REFERENCES}

[1] G. J. R. Povey, P. M. Grant and R. D. Pringle, "A DecisionDirected Spread-Spectrum RAKE Receiver for Fast-Fading Mobile Channels," IEEE Trans. Vehicular Tech., Vol. 45, No. 3, pp. 491-502, August 1996.

[2] W.-H. Sheen and Gordon L. Stuber, "Effects of Multipath Fading on Delay-Locked Loops for Spread Spectrum Systems," IEEE Trans. Commun., Vol. 42, pp. 1947-1956 Feb./March/April 1994.

[3] J. R. Treichler and M. G. Larimore, "New Processing Tech niques Based on the Constant Modulus Adaptive Algorithm," IEEE Trans. Acoust. Speech Signal Process., Vol. ASSP-33, No. 2, pp. 420-431, April, 1985.

[4] John G. Proakis, Digital Communications, NY: MacGraw Hill 1989.

[5] Jia-Chin Lin and Lin-Shan Lee, "A Modified Code Tracking Loop for Direct-Sequence Spread-Spectrum Systems on Frequency-Selective Fading Channels," IEICE Trans. Commun., Vol. E80-B, No. 7, pp. 1055-1063, July 1997.

[6] Jia-Chin Lin and Lin-Shan Lee, "A Modified Blind Equalization Technique Based on a Constant Modulus Algorithm," submitted for review.

[7] K. N. Oh and Y. O. Chin, "New Blind Equalization Techniques Based on Constant Modulus Algorithm," Proc. Globecomm'95, 1995.

[8] O. Shalvi and E. Weinstein, "New Criteria for Blind Deconvolution of Nonminimum Phase Systems (Channels)," IEEE Trans. Inform. Theory, Vol. IT-36, pp. 312-321, Mar. 1990.

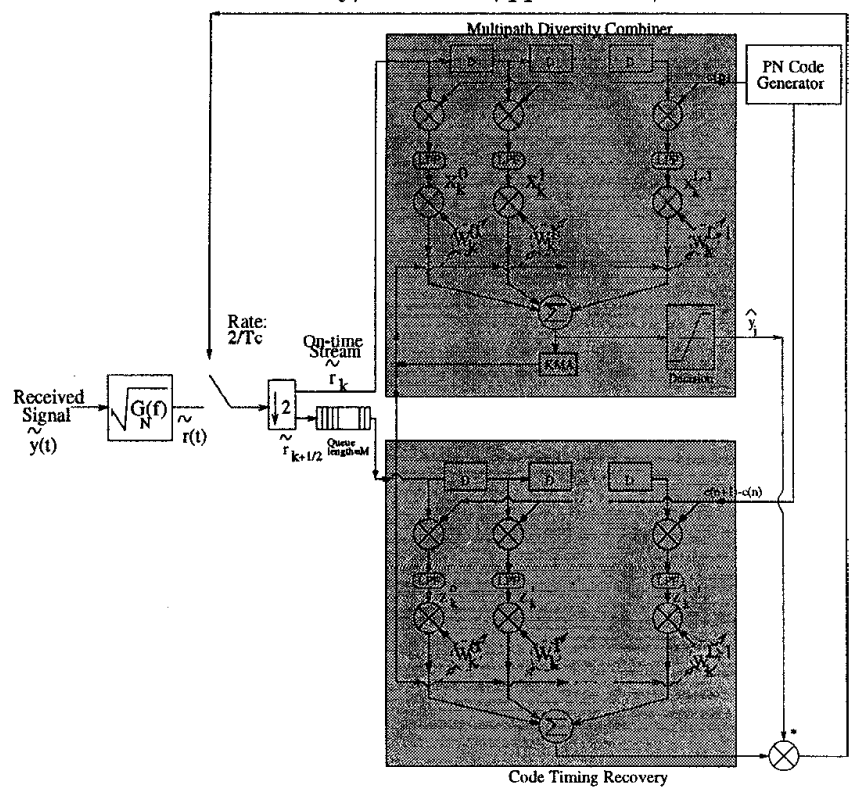

Fig. 1. The RAKE receiver based on the proposed blind joint estimator for multipath diversity and PN code timing error.

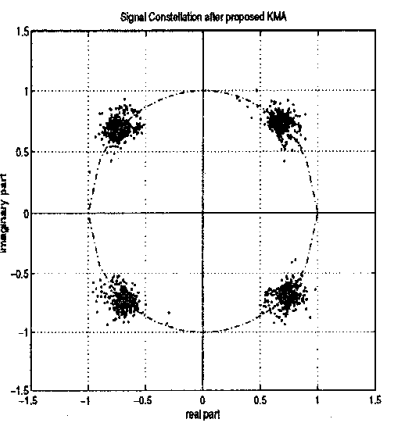

(a)

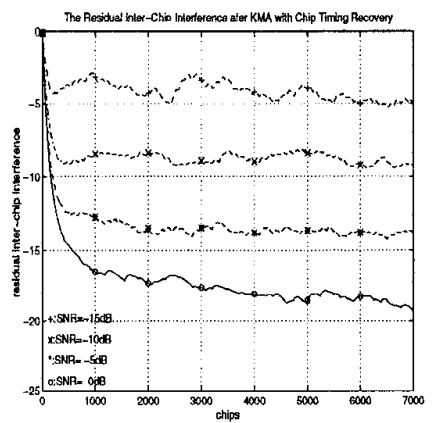

(b)
Fig. 2. (a)Constellation of the output of the KMA-based multipath diversity combiner with $\mathrm{PN}$ code timing recovery.(b)The residual inter-chip interference after $\mathrm{KMA}$ with proposed code timing recovery.

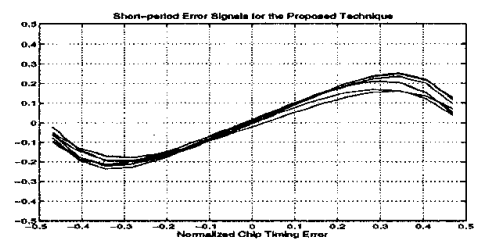

(a)

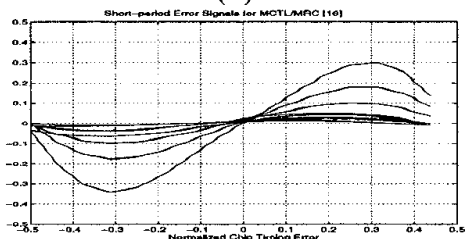

(b)

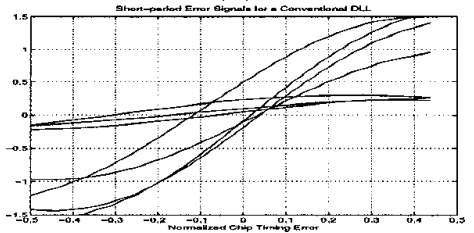

(c)

Fig. 3. (a)several short-period error signals of the proposed code timing recovery, (b)several short-period error signals of MCTL/MRC [5], (c)several short-period exror signals of the conventional DLL.

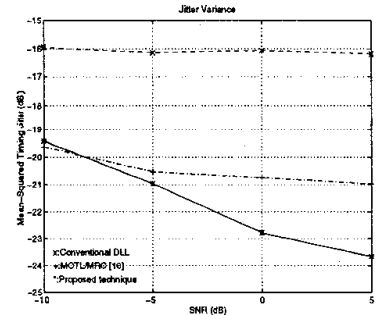

(a)

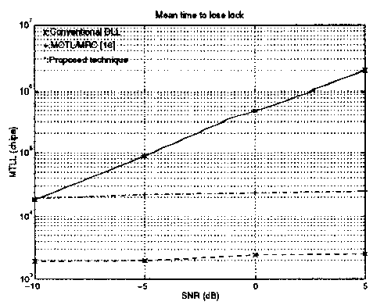

(b)
Fig. 4. (a)Mean squared timing jitter and (b)Mean time to lose lock with (1) x: conventional DLL, (2) +: MCTL/MRC [5], and $(3) *$ : the proposed technique. 